\title{
A finite box as a tool to distinguish free quarks from confinement at high temperatures
}

\author{
L. Ya. Glozman ${ }^{\mathrm{a}}$, C. B. Lang \\ Institute of Physics, University of Graz, 8010 Graz, Austria
}

Received: 22 October 2020 / Accepted: 13 May 2021 / Published online: 5 June 2021

(C) The Author(s) 2021

Communicated by Rishi Sharma

\begin{abstract}
Above the pseudocritical temperature $T_{c}$ of chiral symmetry restoration a chiral spin symmetry (a symmetry of the color charge and of electric confinement) emerges in QCD. This implies that QCD is in a confining mode and there are no free quarks. At the same time correlators of operators constrained by a conserved current behave as if quarks were free. This explains observed fluctuations of conserved charges and the absence of the rho-like structures seen via dileptons. An independent evidence that one is in a confining mode is very welcome. Here we suggest a new tool how to distinguish free quarks from a confining mode. If we put the system into a finite box, then if the quarks are free one necessarily obtains a remarkable diffractive pattern in the propagator of a conserved current. This pattern is clearly seen in a lattice calculation in a finite box and it vanishes in the infinite volume limit as well as in the continuum. In contrast, the full QCD calculations in a finite box show the absence of the diffractive pattern implying that the quarks are confined.
\end{abstract}

\section{Introduction}

At temperatures between 100 and $200 \mathrm{MeV}$ one observes in QCD a smooth chiral symmetry restoration crossover: The quark condensate drops from its practically zero temperature value at $T \sim 100 \mathrm{MeV}$ to the value close to zero at $T \sim 200$ $\mathrm{MeV}[1,2]$. Up to this crossover QCD thermodynamics is well described by a gas of non-interacting mesons. Above the crossover another physics regime emerges that is characterized by a nearly perfect fluidity where there are no free

\footnotetext{
a e-mail: leonid.glozman@uni-graz.at (corresponding author)
}

quarks and gluons and QCD is still in the confining regime. ${ }^{1}$ What are the physical degrees of freedom here and how do they explain that the system is in the liquid regime? Certainly it is not yet a quark-gluon-plasma (QGP) which is a gas and where the degrees of freedom are truly free (i.e., at most weakly interacting, not confined) quarks and gluons.

It is clear from the lattice data for even second order quark and baryon number fluctuations that the free quark gas limit is achieved only at very high temperatures [3]. The continuum extrapolated screening masses for different meson channels agree with the free quark gass estimates only at $T>5 T_{c}$ for scalar-pseudoscalar and at $T>3 T_{c}$ for vector-pseudovector channels [4].

On the other hand there are observables that behave as if quarks were free particles soon above the pseudocritical temperature of chiral symmetry restoration. For example, the ratio of fourth and second cumulants of quark (baryon) number and charge fluctuations approaches a free quark gas value already at $T \sim 200-250 \mathrm{MeV}[3,5]$ and is considered sometimes as "evidence of deconfinement". Another "evidence of deconfinement" is a nonobservation of the $\rho$ like structures via dileptons in experiments. At the same time it was established in lattice calculations of spatial and temporal correlators [6-8] that $\mathrm{QCD}$ in the range $T_{c}-3 T_{c}$ is characterized by the chiral spin symmetry $[9,10]$ which is a symmetry of the color charge and of the chromoelectric interaction. ${ }^{2}$ This is not a symmetry of the Dirac action and hence inconsistent with free interactionless quarks. This suggests that the degrees of freedom are the chirally symmetric quarks bound into the color-singlet objects by the chromo-

\footnotetext{
${ }^{1}$ In QCD with light quarks only one consistent definition of confinement is known: Confinement is the absence of color states in the spectrum. Hence deconfinement should be accompanied by a free motion of colored quarks and gluons.

2 This symmetry was reconstructed from a large hadron spectrum degeneracy observed on the lattice upon artificial subtraction of the near-zero modes of the Dirac operator at zero temperature $[11,12]$.
} 
electric field. What are these objects? ${ }^{3}$ This symmetry was observed in lattice calculations at zero baryon (quark) chemical potential. It should also persist at a nonvanishing chemical potential since the quark chemical potential in the QCD action is manifestly chiral spin symmetric [13].

Both temporal and spatial meson correlators ${ }^{4}$ exhibit this symmetry very clearly at $T_{c}-3 T_{c}$, which suggests a chromoelectric confining interaction. The screening masses extracted from the fit with the proper $\exp (-\mu z) / z$ asymptotics also demonstrate clear multiplets of the chiral spin symmetry below $3 T_{c}$ [16]. At the same time the correlators of operators constrained by a conserved current are very close to those calculated with free quarks [17]. The latter circumstance explains some features of fluctuations of conserved charges above $T_{c}$ and why no $\rho$-like structures are seen via dileptons. This intriguing behavior of the correlators was a motivation for a conjecture of a deconfinement in a $S U(2)_{\text {color }}$ subgroup $^{5}$ of $S U(3)_{\text {color }}$ induced by a $S U(2)_{\text {color }}-S U(2)_{i s o s p i n}$ locking [17]. This would explain both the chiral spin symmetry of the correlators and at the same time their behavior in channels with conserved currents. Because of the $S U(2)_{\text {color }}-S U(2)_{\text {isospin }}$ locking the conserved currents do not see the $S U(3)_{\text {color }} / S U(2)_{\text {color }}$ part of dynamics which is still confining. So while the correlators of the conserved currents behave as if quarks were free, in reality these quarks are still in the confining mode because of the confinement in $S U(3)_{\text {color }} / S U(2)_{\text {color }}$.

Given this intriguing situation an independent evidence is welcome that quarks in channels with conserved currents are still in the confining mode, even though the respective correlators are quite close to those derived with free quarks. This question is the subject of the present paper. We demonstrate that even if the correlators of conserved currents although confined look like those derived for free quarks in the continuum, we can distinguish really free quarks from these freelike behavior by putting the system into a finite box. If quarks are really free, in a finite box this leads to a very specific and bright interference pattern that does not exist in infinite volumes or in the continuum. While we do observe such patterns in a finite box in a free quark system, these patterns are absent in full QCD calculations in a finite box. This allows the conclusion that the quarks are in a confining mode. Hence we have two independent and complementary evidences that QCD is in the confining regime: the chiral spin symmetry of

\footnotetext{
${ }^{3}$ Conditionally the regime in QCD above $T_{c}$ but below $3 T_{c}$ was named a stringy fluid to emphasize the fact that the degrees of freedom are the ultrarelativistic chirally symmetric quarks bound by the chromoelectric field and the chromomagnetic effects are at least strongly suppressed.

${ }^{4}$ Pioneering studies of spatial (screening) propagator are due to Ref. [14]. In [15] lattice screening masses were compared with effective theory approaches.

5 A general possibility of a deconfinement in a $S U(M)$ subgroup of $S U\left(N_{c}\right)$ in different large $N_{c}$ models was discussed in Ref. [18].
}

the correlators and the absence of very pronounced interference patterns required by free quarks in a finite box on the lattice.

\section{Free quarks in a finite box}

In Minkowski space the Feynman propagator of a Dirac particle depending on the chronological order is either a forward running particle $(\sim \exp (-i E t))$ or a backward running antiparticle $(\sim \exp (+i E t))$. Upon a Wick rotation to Euclidean space the forward running particle has an $\sim \exp (-E t)$ dependence while the backward running antiparticle evolves with time as $\sim \exp (+E t)$. If we put the system into a finite box, e.g., on the lattice, then the rest frame $(\mathbf{p}=0)$ time-direction propagator of a free quark with the mass $m$

$C_{0}(t)=\sum_{x, y, z}\langle\psi(x, y, z, t) \bar{\psi}(\mathbf{0}, 0)\rangle$

has a $C_{0}(t) \sim \cosh \left(m\left(t-N_{t} / 2\right)\right)$ dependence for periodic boundary conditions (p.b.c.) along the time direction and a $C_{0}(t) \sim \sinh \left(m\left(t-N_{t} / 2\right)\right)$ form for antiperiodic boundary conditions (a.b.c.). ${ }^{6}$

At nonzero temperature the temporal direction becomes short compared to the spatial one. There are cases in which a study of the propagators along the long spatial direction can supply us with the information that cannot be obtained from the temporal propagators along the short time direction. We choose this direction to be $z$ and study the following spatial correlators:

$C_{S}(z)=\sum_{x, y, t}\langle\psi(x, y, z, t) \bar{\psi}(\mathbf{0}, 0)\rangle$.

This spatial single quark propagator can be straightforwardly calculated on a finite $N_{s}^{3} \times N_{t}$ lattice with given boundary conditions. We choose antiperiodic boundary conditions (a.b.c.) along the time direction, periodic ones (p.b.c.) along the $x, y$ axes and either periodic or antiperiodic along the propagation direction $z$. The results for $\operatorname{Tr} C_{s}(z)$ obtained at zero quark mass with the Wilson and overlap Dirac operators [19] are shown in Fig. 1.

An effective "chirally symmetric mass" $E$ for propagation of a massless quark in $z$ direction is very close to the lowest Matsubara frequency $\pi / N_{t}=\pi / 8$ and is determined by the closest pole position $1 / D\left(p_{x}=0, p_{y}=0, p_{z}=\mathrm{i} E, p_{t}=\right.$ $\left.\pi / N_{t}\right)$ of the Wilson-Dirac operator in momentum space

$$
E=\operatorname{arcosh}\left(\frac{-3+2 \cos \pi / N_{t}}{-2+\cos \pi / N_{t}}\right) \text {. }
$$

\footnotetext{
${ }^{6}$ On a discrete lattice $x, y, z, t$ should be discrete $\left(n_{x}, n_{y}, n_{z}, n_{t}\right) ; N_{t}$ is the lattice size in $t$-direction.
} 


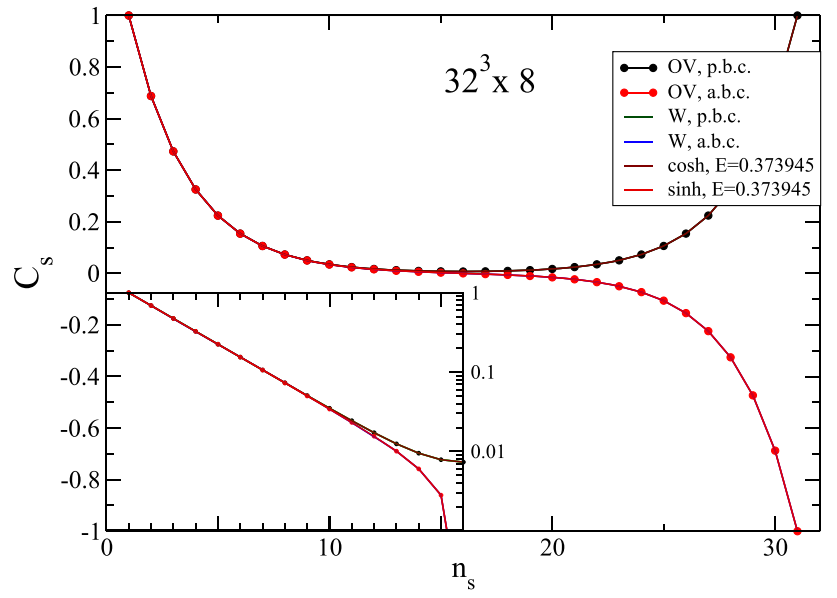

Fig. 1 A single quark massless propagator obtained on the $32^{3} \times 8$ lattice with Wilson and overlap actions in comparison with the $\cosh (E(z-$ $\left.\left.N_{s} / 2\right)\right)$ and $\sinh \left(E\left(z-N_{s} / 2\right)\right)$ at $E=0.37395$. Note that propagators obtained with Wilson and overlap actions coincide within 3 digits and cannot be distinguished in the plot

The propagator obtained for a single quark with Wilson or overlap Dirac action is very accurately described by $\cosh \left(E\left(z-N_{s} / 2\right)\right)$ for p.b.c. and by $\sinh \left(E\left(z-N_{s} / 2\right)\right)$ for a.b.c.. This propagator can be interpreted as a superposition of a forward $(f)$ running quark with the "mass" $E$ and of a backward $(b)$ running antiquark with the same "mass". Symbolically the propagator can be written as

$C_{S}(z)^{p . b . c .} \sim \exp (-E z)+\exp \left(-E\left(N_{s}-z\right)\right) \equiv f+\bar{b}$.

For the a.b.c. the propagator is

$C_{S}(z)^{a . b . c .} \sim \exp (-E z)-\exp \left(-E\left(N_{s}-z\right)\right) \equiv f-\bar{b}$.

Having discussed the structure of a single quark propagator in a finite box we next study propagators of quark bilinears still keeping quarks to be noninteracting particles (I.e., due to a pure Dirac Lagrangian without any gauge fields.) The spatial correlators of the isovector bilinear operators $\mathcal{O}_{\Gamma}(x, y, z, t)=\bar{\psi}(x, y, z, t) \Gamma \frac{\tau}{2} \psi(x, y, z, t)$ with $\Gamma$ being out of a set of $\gamma$-matrices are

$C_{\Gamma}(z)=\sum_{x, y, t}\left\langle\mathcal{O}_{\Gamma}(x, y, z, t) \mathcal{O}_{\Gamma}(\mathbf{0}, 0)^{\dagger}\right\rangle$.

The isovector fermion bilinears are named according to Table 1.

A complete set of such propagators in the continuum (in infinite volume) has been determined analytically in Ref. [7]. There these correlators are given as superpositions of the decaying exponents $\exp \left(-2 \pi n_{z} / N_{t}\right) /\left(2 \pi n_{z} / N_{t}\right)$, $\exp \left(-2 \pi n_{z} / N_{t}\right) /\left(2 \pi n_{z} / N_{t}\right)^{2}, \ldots$ and terms with higher Matsubara frequencies and represent the propagators of the forward propagating "mesons" that are made from noninteracting quarks.
Table 1 Fermion isovector bilinears and their $U(1)_{A}$ and $S U(2)_{L} \times$ $S U(2)_{R}$ transformation properties (last column). This classification assumes propagation in $z$-direction. The open vector index $k$ here runs over the components $1,2,4$, i.e., $x, y$ and $t$

\begin{tabular}{llll}
\hline Name & Dirac structure $\Gamma$ & Abbreviation & \\
\hline Pseudoscalar & $\gamma_{5}$ & $P S$ & ]$U(1)_{A}$ \\
Scalar & $\mathbb{1}$ & $S$ & \\
Axial-vector & $\gamma_{k} \gamma_{5}$ & $\mathbf{A}$ & ]$S U(2)_{A}$ \\
Vector & $\gamma_{k}$ & $\mathbf{V}$ & \\
Tensor-vector & $\gamma_{k} \gamma_{3}$ & $\mathbf{T}$ & ]$U(1)_{A}$ \\
Axial-tensor-vector & $\gamma_{k} \gamma_{3} \gamma_{5}$ & $\mathbf{X}$ & \\
\hline
\end{tabular}

In a finite box a quark propagator of a given flavor is represented as a sum (for p.b.c) or difference (for a.b.c.) of the forward propagating quark and of the backward propagating antiquark. The same is true for the antiquark propagator, that is a sum (or difference) of the forward propagating antiquark and of the backward propagating quark. Consequently correlators of the bilinears should be superpositions of four terms:

$$
\begin{aligned}
& \text { p.b.c. : } \quad(f+\bar{b})(\bar{f}+b)=f \bar{f}+b \bar{b}+f b+\bar{b} \bar{f}, \\
& \text { a.b.c. : } \quad(f-\bar{b})(\bar{f}-b)=f \bar{f}+b \bar{b}-f b-\bar{b} \bar{f} .
\end{aligned}
$$

Note that the two terms $\sim \bar{f} f$ and $\sim \bar{b} b$ represent the forward and backward propagating meson-like system. The other two terms $\sim f b$ and $\sim \bar{f} \bar{b}$ do not represent any meson-like system. More precisely, they represent a quark-antiquark system where the quark and the antiquark are shifted relative to each other by a large distance $N_{s}$. These terms are necessarily present in the correlators of the quark-antiquark bilinears if quarks are free particles that do not interact. They exist only in a finite box and vanish in the infinite volume limit or in the physical continuum. If we put the system of free quarks into a finite box, then these "unphysical" terms must be observable since they interfere with the "physical" meson-like amplitudes. The interference should be clearly seen in cases when the "physical" and "unphysical" terms are of a similar magnitude and interfere destructively. Since the "unphysical" terms are very small one should expect this destructive interference to be clearly visible only when the "physical" terms are also very small. The numerical results for the propagators calculated with free noninteracting quarks [6] show that the largest slope of the decay takes place with the operators $V_{t}, A_{t}, T_{x}, T_{y}, X_{x}, X_{y}$ and all other operators $V_{x}, V_{y}, A_{x}, A_{y}, \ldots$ have smaller decay rate. $^{7}$ This suggests that the "physical" meson-like amplitude becomes sufficiently small at large $z$ for the operators $V_{t}, A_{t}, T_{x}, T_{y}, X_{x}, X_{y}$ and we can expect in this case well

\footnotetext{
$7 V_{t}$ refers to the $\bar{\psi}(x, y, z, t) \gamma_{4} \frac{\tau}{2} \psi(x, y, z, t)$ operator, etc, see the legend to the table.
} 

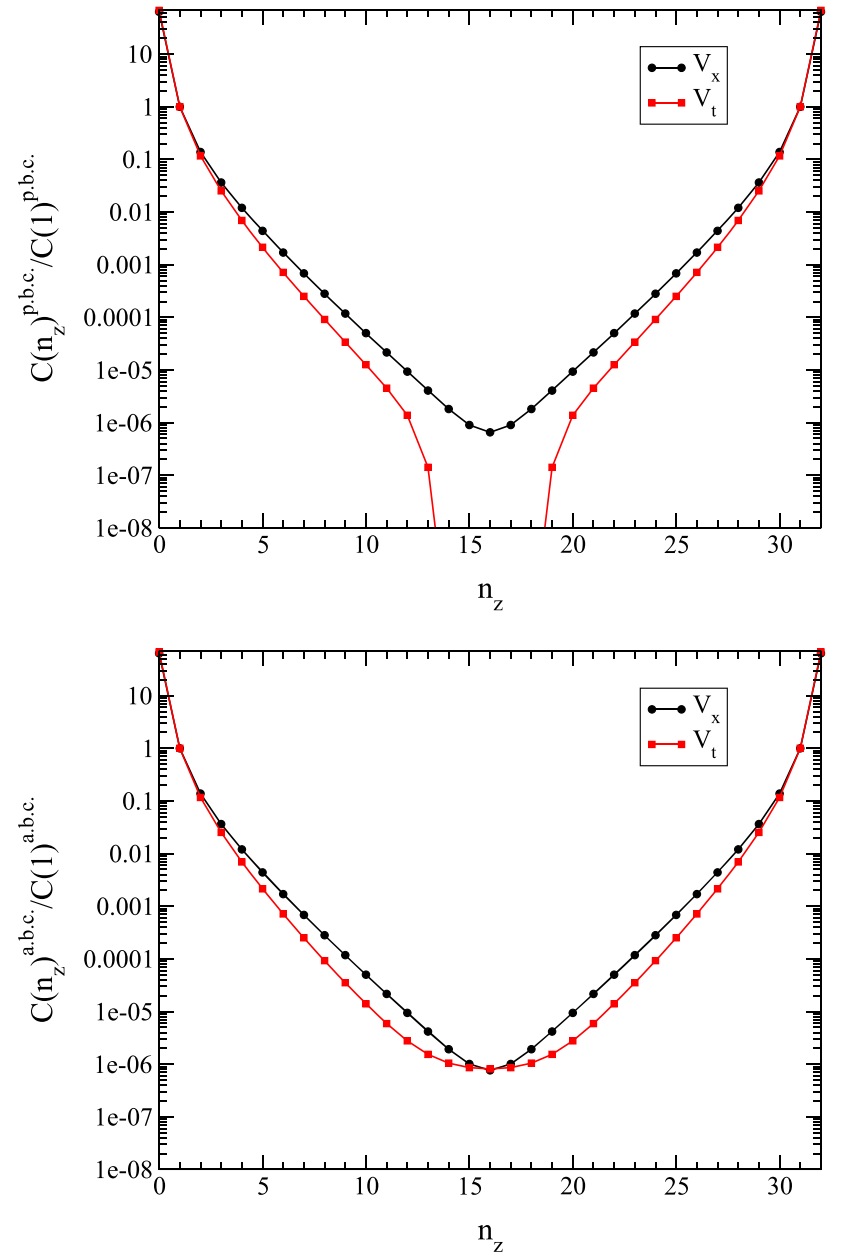

Fig. 2 Correlators of the $V_{t}$ and $V_{x}$ bilinears on a $32^{3} \times 8$ lattice with the overlap action with periodic (p.b.c., left panel) and antiperiodic (a.b.c., right panel) boundary conditions in $z$ direction. The $V_{t}$ correlator in the left panel is negative for $n_{z} \sim 14-18$. The correlators are normalized to 1 at $n_{z}=1$

visible interference effects of the "physical" and "unphysical" amplitudes.

The correlators calculated with the overlap action on the $32^{3} \times 8$ lattice with the $V_{t}$ operator are shown in Fig. 2. The correlators of the $A_{t}, T_{x}, T_{y}, X_{x}, X_{y}$ operators are similar. We also show in the same figure the correlators of the $V_{x}$ operator that demonstrates a smaller decay rate.

We clearly see a typical diffractive structure for the correlator of the $V_{t}$ operator at large $z$ and when p.b.c. are imposed the correlator becomes negative for $z \sim 14-18$. This was first noted in Refs. $[6,20]$ but remained unexplained. Now we realize that this structure is the result of the destructive interference of the "physical" and "unphysical" amplitudes. It is an immanent property of a system of free quarks in a finite box. In contrast, the correlator of the $V_{x}$ operator does not show a diffractive structure because the "physical" terms in this case are always essentially larger than the "unphysical" ones.
How to check this picture of the destructive interference? If we change from p.b.c. to a.b.c. one should expect a constructive interference of the "physical" and "unphysical" terms. Hence the diffractive structure should disappear. This is precisely what happens.

Numerical checks indicate that the diffractive structure disappears exponentially upon increase of $N_{s}$ (at fixed $N_{t}$ ). Hence it vanishes both in large lattice volumes as well as in the continuum theory.

\section{Comparison of the full QCD and free quarks correlators in a finite box}

We have established in the previous section that if quarks are free, then the spatial correlators of the conserved currents $V_{t}, A_{t}$ and of some other operators exhibit on a finite lattice remarkable diffractive patterns. These are a consequence of the fact that for free quarks there are necessarily amplitudes that represent a "meson-like" propagation, called "physical", and "unphysical" amplitudes that do not correspond to any meson-like system. These "physical" and "unphysical" amplitudes interfere destructively. The "unphysical" amplitudes vanish on the infinite lattice as well as in the continuum and the diffractive pattern disappears.

At the same time the "unphysical" terms are much smaller than the "physical" ones for another set of operators and the diffractive pattern does not exist. These features are a solid prediction of a free quark system put on a finite lattice.

In the continuum full QCD above $T_{c}$ the spatial and temporal correlators of the conserved currents are close to those ones calculated with noninteracting quarks [17]. In reality quarks cannot be free since these correlators are subject to the chiral spin symmetry that is not a symmetry of the Dirac action. Is there another means to decide that the quarks are not free? The answer is affirmative. When we solve QCD at high temperatures on the finite lattice if the quarks are not confined (i.e., free), one should observe the diffractive pattern as described above. If such a pattern is missing, then we could safely conclude that the quarks are not free. This is demonstrated below.

In Fig. 3 we show correlators normalized to 1 at $n_{z}=1$ built with the $V_{t}, A_{t}, T_{x}, X_{x}$ operators calculated in $N_{F}=2$ QCD with the domain wall Dirac operator at physical quark masses on $32^{3} \times 8$ lattice at $T=380 \mathrm{MeV}\left(2.2 T_{c}\right)$ [6]. The boundary conditions for quarks are a.b.c. in time direction and p.b.c. in all spatial directions. The solid curves represent the full QCD results while the dashed curves are correlators calculated on the same lattice with the same Dirac operator with free noninteracting quarks, i.e. computed with a trivial gauge field configuration $(U=1)$. The free quark correlator of the $V_{t}$ operator corresponds to the results shown in Fig. 2. It is rather obvious that the free quark results obtained with 


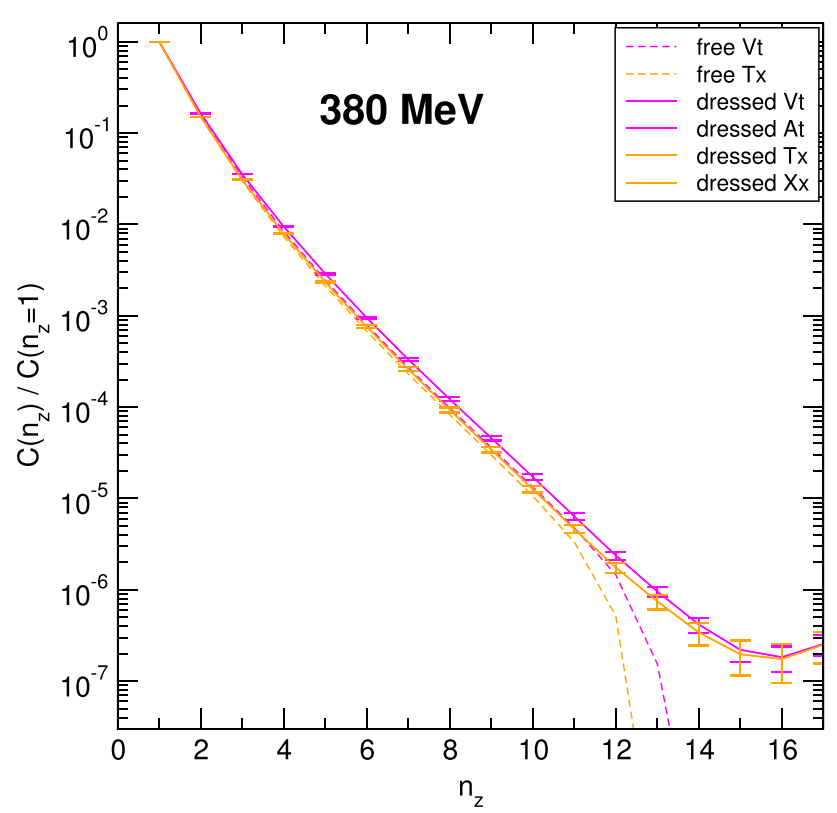

Fig. 3 Correlators of the $V_{t}, A_{t}, T_{x}, X_{x}$ operators in full QCD at $T=$ $380 \mathrm{MeV}\left(\sim 2.2 T_{c}\right)$ for $32^{3} \times 8$ lattice (abbreviated as dressed) and with non-interacting quarks (free) on the same lattice. From Ref. [6]

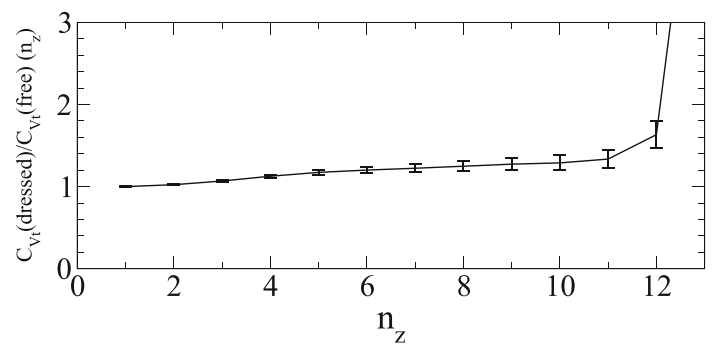

Fig. 4 Ratio of correlator of $V_{t}$ operator in full QCD at $T=380 \mathrm{MeV}$ $\left(\sim 2.2 T_{c}\right.$ ) for $32^{3} \times 8$ lattice (abbreviated as dressed) to $V_{t}$ correlator calculated with non-interacting quarks on the same lattice size (free)

the domain wall Dirac operator in Fig. 3 are similar to those obtained with the overlap Dirac operator in Fig. 2. In both cases we see a remarkable diffractive structure around $n_{z} \sim$ $12-20$.

This diffractive structure is induced via a destructive interference of the "physical" meson-like amplitudes with the "unphysical" amplitudes that do not correspond to any meson-like system. This structure necessarily exists on a finite lattice if the quarks are free noninteracting particles. In contrast, the full QCD results do not show this diffractive pattern. The diffractive pattern is induced by the deep infrared region, i.e., by large distances between quarks. Even though the propagator of the conserved current $V_{t}$ in full QCD is rather close to the free quarks propagator at $n_{z}<11$, for the ratio of both propagators see Fig. 4, it does not represent a free quark system but describes a propagation of a meson-like system with confinement. All "unphysical" terms that exist in the case of the free quark system are killed by a confining

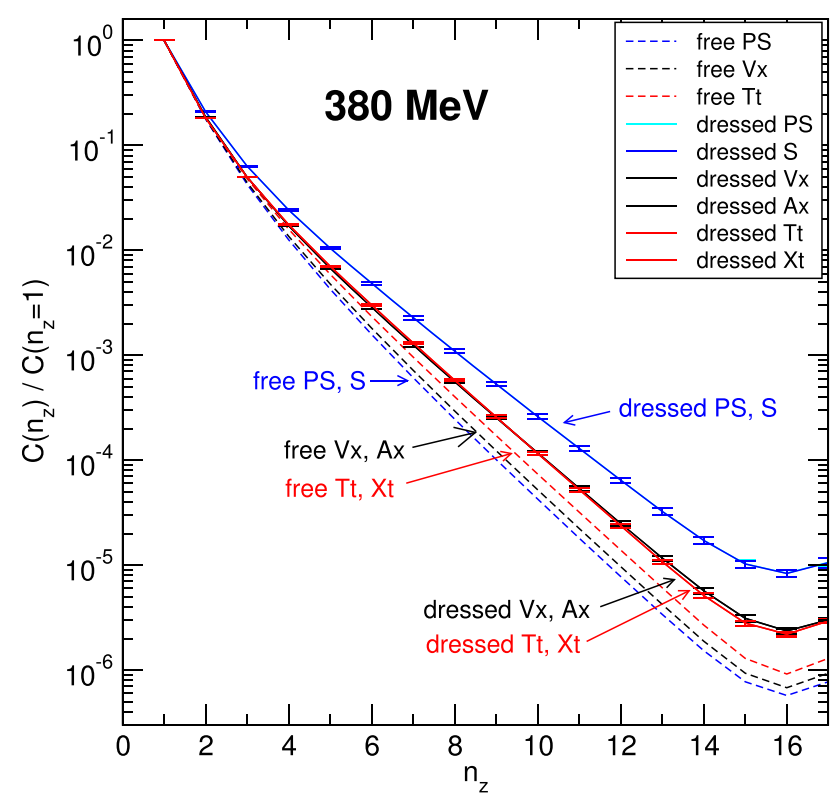

Fig. 5 Correlators of the $P S, S, V_{x}, A_{x}, T_{t}, X_{t}$ operators in full QCD at $T=380 \mathrm{MeV}\left(\sim 2.2 T_{c}\right)$ for $32^{3} \times 8$ lattice (abbreviated as dressed) and with non-interacting quarks (free) on the same lattice. From Ref. [6]

gluonic interaction between quarks that are separated by a large spatial distance. The fluctuations of conserved charges are given by integrals of the correlator. Since the crucial deviations of the free quark correlator from the QCD correlator are seen only at very large distances (where the absolute value of the correlators is suppressed by 5-6 orders of magnitude) a sensitivity of the fluctuations of the conserved charge to the deep infrared is only weak.

The propagator of the $V_{x}$ operator, that is not constrained by a current conservation, demonstrates the absence of the diffractive structure both in full QCD as well as for free quarks, see Fig. 5.

We summarize this section with the principal result of the present paper. There are two independent evidences that a system with quantum numbers of a conserved current is in a confining mode above $T_{c}$. The first evidence are the very clear patterns of the chiral spin symmetry both in spatial and temporal correlators [6-8]. The second evidence, demonstrated in the present paper, is the absence of the diffractive pattern required by a system of free quarks.

\section{Discussion and conclusions}

We have demonstrated that on a finite lattice in a system of free noninteracting quarks the spatial propagators of the bilinear quark-antiquark operators exhibit in case of periodic boundary conditions along the propagation direction a diffractive pattern for operators that are constrained by a cur- 
rent conservation and for some other operators. This diffractive pattern is a consequence of a destructive interference of the amplitudes that correspond to the propagation of a meson-like system made of a quark and an antiquark with amplitudes that do not describe any meson-like system. The latter amplitudes arise exclusively due to a finiteness of a box and vanish on an infinite lattice or in the continuum. The latter amplitudes as well as a diffractive pattern is an immanent property of the free quark system in a finite lattice.

In QCD the correlators of conserved currents above a chiral symmetry restoration crossover behave as if quarks were free, i.e. the correlators of these currents calculated in QCD are rather close to correlators obtained with free noninteracting quarks [17]. This explains why some cumulants of fluctuations of conserved charges suggest a free quark gaslike behavior very soon above $T_{c}$ as well as absence of the rho-like structures observed via dileptons in heavy ion collisions. At the same time these correlators as well as another ones are a subject to a chiral spin symmetry $[9,10]$ at $T_{c}-3 T_{c}$ [6-8]. This symmetry is not a symmetry of the Dirac action and hence inconsistent with free noninteracting quarks. It is a symmetry of the color charge in QCD and it indicates that QCD is in the confining regime where the chromoelectric interaction binds the chirally symmetric quarks into colorsinglet objects ("strings") and a contribution of the chromomagnetic interaction is at least strongly suppressed.

An independent evidence confirming that the quarkantiquark systems with a conserved current quantum numbers are indeed in the confining regime is supplied by QCD on the lattice in a finite box. If the quarks are free, then there must be a diffractive pattern described above that is induced by quarks that are separated by a large space distance. In full QCD calculations above $T_{c}$ in a finite box such pattern is not observed. It follows then that the quarks are not free and confining chromoelectric dynamics kills all amplitudes that do not correspond to propagating mesons.

Hence we have two independent and complementary evidences of confinement in at $T_{c}-3 T_{c}$. These are the chiral spin symmetry of correlators and the absence of a diffractive structure required by free quarks in a finite box. This regime we have conditionally called "stringy fluid" $[7,13]$.

At temperatures above $3 T_{c}$ the chiral spin symmetry smoothly disappears [7] and correlators of all operators approach correlators calculated with free quarks. This suggests that eventually the color charge and electric confining interaction is Debye screened within $S U(3)_{\text {color }}$ (cf., Ref. $[21,22])$. Still the correlators of the conserved currents in a finite box do not show the diffractive structure required by really free quarks [7]. This indicates that there are no free, noninteracting quarks and the system is still in the confining regime (defining confinement as the absence of free quarks and gluons.) The latter fact can be explained by the presence of a weak magnetic confinement at very high temperatures.
It is known that at very high temperatures QCD is dimensionally reduced to a weakly coupled 3-dimensional pure magnetic theory [23]. Even though the theory is weakly coupled, there is a pure magnetic weak "confining" interaction that does not allow quarks to be completely free $[21,24,25]$. In this regime all properties of QCD should be close to the quark-gluon-plasma regime.

We emphasize that the absence of a diffractive pattern in full QCD spatial correlators evidences confinement, but it cannot distinguish between the electric and magnetic confinement. Only the chiral spin symmetry observed in the range $T_{c}-3 T_{c}[7,8]$ does suggest that it is an electric confinement that drives properties of QCD in the stringy fluid regime.

Acknowledgements We thank T. Cohen, C. Gattringer, O. Philipsen and R. Pisarski for careful reading of the ms.

Funding Open access funding provided by University of Graz.

Data Availability Statement This manuscript has no associated data or the data will not be deposited. [Authors' comment: All data generated in this study are contained in the published article.]

Open Access This article is licensed under a Creative Commons Attribution 4.0 International License, which permits use, sharing, adaptation, distribution and reproduction in any medium or format, as long as you give appropriate credit to the original author(s) and the source, provide a link to the Creative Commons licence, and indicate if changes were made. The images or other third party material in this article are included in the article's Creative Commons licence, unless indicated otherwise in a credit line to the material. If material is not included in the article's Creative Commons licence and your intended use is not permitted by statutory regulation or exceeds the permitted use, you will need to obtain permission directly from the copyright holder. To view a copy of this licence, visit http://creativecomm ons.org/licenses/by/4.0/.

\section{References}

1. Y. Aoki, S. Borsanyi, S. Durr, Z. Fodor, S.D. Katz, S. Krieg, K.K. Szabo, JHEP 0906, 088 (2009)

2. A. Bazavov et al. [HotQCD Collaboration], Phys. Lett. B 795, 15 (2019)

3. A. Bazavov et al., Phys. Rev. D 95(5), 054504 (2017)

4. A. Bazavov et al., Phys. Rev. D 100 (2019) no.9, 094510 https://doi. org/10.1103/PhysRevD.100.094510. arXiv:1908.09552 [hep-lat]

5. F. Karsch, S. Ejiri, K. Redlich, Nucl. Phys. A 774, 619 (2006)

6. C. Rohrhofer, Y. Aoki, G. Cossu, H. Fukaya, L.Y. Glozman, S. Hashimoto, C.B. Lang, S. Prelovsek, Phys. Rev. D 96, 094501 (2017). Erratum: [Phys. Rev. D 99, 039901 (2019)]

7. C. Rohrhofer, Y. Aoki, G. Cossu, H. Fukaya, C. Gattringer, L.Y. Glozman, S. Hashimoto, C.B. Lang, S. Prelovsek, Phys. Rev. D 100, 014502 (2019)

8. C. Rohrhofer, Y. Aoki, L.Y. Glozman, S. Hashimoto, Phys. Lett. B 802, 135245 (2020)

9. L.Y. Glozman, Eur. Phys. J. A 51, 27 (2015)

10. L.Y. Glozman, M. Pak, Phys. Rev. D 92, 016001 (2015)

11. M. Denissenya, L.Y. Glozman, C.B. Lang, Phys. Rev. D 89, 077502 (2014) 
12. M. Denissenya, L.Y. Glozman, C.B. Lang, Phys. Rev. D 91, 034505 (2015)

13. L.Y. Glozman, Eur. Phys. J. A 54, 117 (2018)

14. C.E. Detar, J.B. Kogut, Phys. Rev. Lett. 59, 399 (1987)

15. B.B. Brandt, A. Francis, M. Laine, H.B. Meyer, JHEP 05, 117 (2014)

16. C. Rohrhofer et al, will be released soon; for preliminary results see APLAT 2020 contribution https://conference-indico.kek.jp/event/ 113/contributions/2162/

17. L.Y. Glozman, Phys. Rev. D 102, 014509 (2020)

18. M. Hanada, G. Ishiki, H. Watanabe, JHEP 1903, 145 (2019). Erratum: [JHEP 1910, 029 (2019)]
19. C. Gattringer, C.B. Lang, Quantum chromodynamics on the lattice. Lect. Notes. Phys. 788, 1 (2010)

20. R.V. Gavai, S. Gupta, R. Lacaze, Phys. Rev. D 78, 014502 (2008)

21. V. Koch, E.V. Shuryak, G.E. Brown, A.D. Jackson, Phys. Rev. D 46, 3169 (1992). Erratum: [Phys. Rev. D 47, 2157 (1993)]

22. K. Kajantie, M. Laine, K. Rummukainen, Y. Schroder, Phys. Rev. D 67, 105008 (2003)

23. T. Appelquist, R.D. Pisarski, Phys. Rev. D 23, 2305 (1981)

24. A.D. Linde, Phys. Lett. B 96, 289 (1980)

25. A. Hart, M. Laine, O. Philipsen, Nucl. Phys. B 586, 443 (2000) 